\title{
CYTOKINE PRODUCTION IN EXPERIMENTAL PARACOCCIDIOIDOMYCOSIS OF MURINE SELECTED LINEAGES FOR ACUTE INFLAMMATORY RESPONSE
} (AIR)

Thesis: J. S. Cavalheiro submitted this dissertation for her Doctorate in Tropical Diseases at the Botucatu Medical School, São Paulo State University, UNESP, Botucatu, São Paulo State, Brazil, 2009.

Advisor: Professor Silvio Luis de Oliveira

ABSTRACT: Paracoccidioidomycosis is a systemic human mycosis caused by Paracoccidioides brasiliensis ( $P$. brasiliensis), an imperfect dimorphic fungus whose conidia are its infective form. Mice genetically selected for maximum (AIRmax) and minimum (AIRmin) acute inflammatory response were used as experimental paracoccidioidomycosis models. The animals were intraperitoneally inoculated with P. brasiliensis (strain 18) and killed 6, 12 and 24 hours or 3,7 and 14 days after infection. In these periods, fragments from their spleen, liver and lung were removed for evaluation of the infection level by fungal cells, assessment of macrophagic activity by peritoneal and splenic macrophages - through the determination of nitric oxide (NO) concentrations and production of pro- and anti-inflammatory cytokines of lung and spleen homogenate supernatants. In the present study, it was observed that AIRmax lineages presented greater control of the infectious process than the AIRmin ones. Regarding NO production, AIRmax animals produced more metabolites in late periods, what may help control the infectious process. Concerning cytokine production, it was observed that the production of INF-gamma, TNF-alpha, IL-1, IL-6, IL-8 and IL-12 were increased in AIRmax lineages in most analyzed organs and periods, thus contributing to the greater resistance exhibited by such lineages against infection, except for IL-4 and IL-10 that showed decreased production in AIRmax lineage, reproducing its suppressive biological effect. From these results, it was observed that the AIRmax lineage was more effective in controlling the infectious process, with an important involvement of the analyzed cytokines. These findings are probably related to the genetically selected factors involved in the acute inflammatory response.

KEY WORDS: immune response, Paracoccidioides brasiliensis, cytokines, acute inflammatory response.

\section{CORRESPONCE TO:}

JULIANA SEMIM CAVALHEIRO, Departamento de Microbiologia e Imunologia, Instituto de Biociências, UNESP, Botucatu, SP, Brasil. Phone: +55 14 3811-6058. Email: jscvet@bol.com.brl jusemim@gmail.com. 